\title{
Pituitary intratumoral hemorrhage during radiation therapy following partial removal of giant pituitary adenoma: A case report
}

\author{
Junyang Liu ${ }^{1}$, Yuichiro Yoneoka ${ }^{2}$, Kensuke Tanaka $^{1}$, Hiraku Satou ${ }^{1}$, Eisuke Abe ${ }^{1}$, \\ Naoto Watanabe ${ }^{2}$, Yukihiko Fuji ${ }^{2}$, Hitoshi Takahashi ${ }^{3}$, Hidefumi Aoyama ${ }^{1 *}$ \\ ${ }^{1}$ Department of Radiology, Niigata University Graduate School of Medical and Dental Sciences, Niigata, Japan; \\ ${ }^{*}$ Corresponding Author: h-aoyama@med.niigata-u.ac.jp \\ ${ }^{2}$ Department of Neurosurgery, Brain Research Institute, Niigata University, Niigata, Japan \\ ${ }^{3}$ Department of Pathology, Brain Research Institute, Niigata University, Niigata, Japan
}

Received 13 November 2013; revised 10 December 2013; accepted 30 December 2013

Copyright (C) 2014 Junyang Liu et al. This is an open access article distributed under the Creative Commons Attribution License, which permits unrestricted use, distribution, and reproduction in any medium, provided the original work is properly cited. In accordance of the Creative Commons Attribution License all Copyrights @ 2014 are reserved for SCIRP and the owner of the intellectual property Junyang Liu et al. All Copyright (C) 2014 are guarded by law and by SCIRP as a guardian.

\section{ABSTRACT}

We report a rare case of intratumoral hemorrhage during postoperative radiotherapy for pituitary adenoma. A 57-year-old Asian male, complaining of long-standing eye strain, underwent a medical checkup of the brain. Magnetic resonance imaging showed a multicystic giant pituitary adenoma. The patient underwent an endoscopic endonasal transsphenoidal partial removal of the adenoma to provide optic pathway decompression and got relief from the visual symptoms. Just before completion of the postoperative radiotherapy for residual adenoma, the patient developed right hemiparesis, mild motor aphasia, and right oculomotor palsy. A cranial CT scan showed intratumoral hemorrhage into the intratumoral cyst. The patient therefore had to undergo three additional craniotomies for evacuation of cyst contents over the next 8 months. The follow-up MRI at 11 months after the initial hemorrhage showed that the new oozing of blood in the intratumoral cyst was still appearing. Intratumoral hemorrhage is a rare, albeit life-threatening, complication of pituitary adenoma. We reviewed relevant literature and suggested that the cystic component in pituitary adenoma could be a key pathogenesis of this rare complication. In conclusion, we suggest that it may be necessary to realize that cases which have cystic giant pituitary adenoma may cause hemorrhage by chance with the foreseeability.

\section{KEYWORDS}

\section{Pituitary Hemorrhage; Pituitary Adenoma;} Radiotherapy; Cyst; Giant

\section{INTRODUCTION}

Pituitary hemorrhage is a life-threatening clinical condition caused by rapid enlargement of pituitary tumors due to hemorrhage and/or infarction. It is not fully understood if radiation could be a cause of hemorrhage. We report a case of pituitary intratumoral hemorrhage that occurred during radiation therapy for a residual tumor of giant pituitary adenoma after partial surgical resection. The causal relationship between radiation and hemorrhage is discussed through a relevant literature review. The relationship between cysts and hemorrhage is also addressed.

\section{CASE REPORT}

A 57-year-old man, complaining of long-standing eye-strain, was incidentally diagnosed as having a giant pituitary adenoma $(>4 \mathrm{~cm}$ ) based on a routine magnetic resonance imaging (MRI) scan. Cranial MRI revealed a pituitary adenoma $59 \mathrm{~mm}$ in one extension and the existence of multiple cysts without fluid-fluid level formation (Figure 1(A)). The patient underwent an intentional partial removal through a transsphenoidal approach in order to obtain optic pathway decompression, which resulted in an improvement of the visual field but with slight oculomotor paresis remaining (Figure 1(B)). Radiation of 45 Gy in 25 fractions was administered for sta- 
bilization of the residual tumor starting on the $82^{\text {nd }}$ postoperative day. The patient developed oculomotor palsy at 37.8 Gy (21 fractions). MRI revealed tumor hemorrhage into the preexisting cyst and formed fluid-fluid level (Figure 1(C)). Since medical treatment failed to control the intratumoral oozing of blood and the patient's symptoms gradually worsened, the patient underwent transcranial surgery (TCS) as a second operation 25 days after the onset of intratumoral hemorrhage. Post-TCS MRI showed intratumoral hemorrhage (Figure 1(D)). With his aphasia improved, the intratumoral oozing of blood became symptomatic again 3.5 months after the second operation. The patient underwent TCS as a third operation. However, 7 months after the initial hemorrhage into the adenoma, his aphasia and paralysis worsened again. MRI/CT scan showed that the cyst grew to a larger size with an intra-cystic clot (Figure 1(E)). Although TCS as the fourth operation was done after 2 weeks of medical treatment, his aphasia remained even after surgery. The follow-up MRI at 11 months after the initial hemorrhage showed new oozing of blood in the intratumoral cyst.

\section{HISTOPATHOLOGICAL FINDINGS}

Evaluable pathological specimens were obtained in the first three of the four total operations. Hematoxylin-eosin (HE) staining showed chromophobe adenoma-containing cells with a regular, spherical nucleus and a rather abundant, poorly staining cytoplasm. Immunohistochemical staining, including Ki-67/MIB-1 and vascular endothelial growth factor (VEGF) showed no over expression at baseline and no significant differences among the specimens obtained in those three operations (Figure 2).

\section{DISCUSSION}

Pituitary tumor hemorrhage in the acute phase of radia-
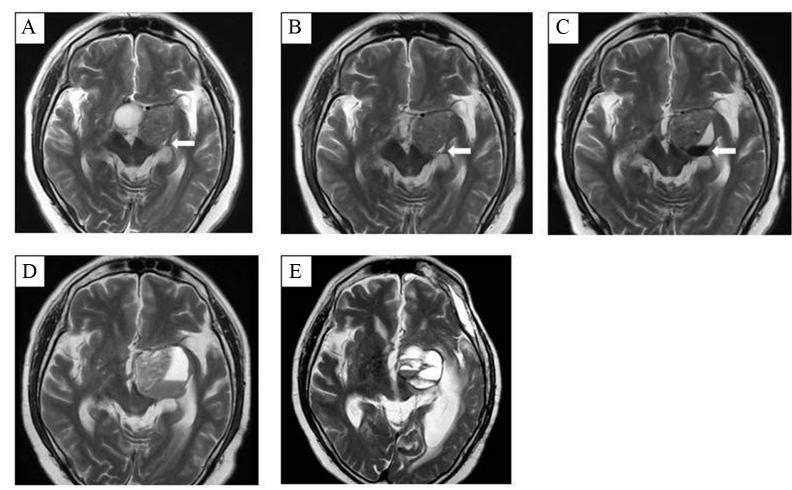

Figure 1. MRI revealed tumor hemorrhage into the preexisted cyst and fluid-fluid level formation. (A) Pre-operation. (B) Pre-radiotherapy. (C) One week since hemorrhage appeared. (D) One month since hemorrhage appeared. (E) Seven months since hemorrhage. tion therapy is extremely rare. Grigsby's series published in 1988 [1] consisted of 70 patients with pituitary adenoma treated with radiation therapy alone; 2 tumor hemorrhages at $1230 \mathrm{cGy}$ and 2239 cGy were observed. Other than that, including abundant modern radiosurgical series, tumor hemorrhage in the acute phase of radiation therapy has rarely been reported. It is not fully understood if radiation could be a direct cause of tumor hemorrhage. Older series, including Grigsby's have suggested a causal effect of radiation on tumor hemorrhage $[1,2]$. However, a more recent series does not suggest such causality [3]. One possible explanation for the causal effect of radiation on pituitary tumor hemorrhage is that radiation therapy may increase the vascularization of pituitary adenomas. This can be shown either angiographically by an enhanced tumor blush or histologically by vessel proliferation with associated thickening and hyaline degeneration of the vessel wall [4]. Another explanation is that the changes in vascular flow after irradiation result in chronic hypoperfusion of the pituitary gland and have been associated with pituitary infarction or hemorrhage [3,5]. In relation to the hypoperfusion theory; Lee JS et al. have reported a positive correlation of VEGF with both radiological and non-radiological apoplexy [3]. This finding suggests that radiation-induced tumor hypoxia could be a cause of subsequent tumor hemorrhage [6]. However overexpression of VEGF at baseline was not observed in the current case and the expression status did not change after the postradiation phase. This theory therefore could not be applied to the current case. Liu $\mathrm{ZH}$ et al. have reported survey results regarding predisposing factors for pituitary hemorrhage, indicating a significant association with macroadenoma, non-functioning adenoma, anticoagulation therapy, end-stage renal disease, dopamine agonist treatment, and underlying malignant disease, but no significant relationship between radiation therapy and

\begin{tabular}{|c|c|c|c|}
\hline & $\mathrm{HE}$ & MIB-I & VEGF \\
\hline $\begin{array}{c}\text { FIRST } \\
\text { OPERATION }\end{array}$ & & & \\
\hline $\begin{array}{l}\text { SECOND } \\
\text { OPERATION }\end{array}$ & & & \\
\hline $\begin{array}{c}\text { THIRD } \\
\text { OPERATION }\end{array}$ & & & \\
\hline
\end{tabular}

Figure 2. Histopathological findingsof three operations (Original magnification $\times 200$ ): Immunohistochemical staining, including Ki-67/MIB-1, vascular endothelial growth factor (VEGF), showed no significance differences among the specimens obtained in those three operations. 
pituitary hemorrhage [4]. Of the above factors, only two, macroadenoma and non-functioning adenoma fit the current case. Regarding the effect of tumor size on the risk of tumor hemorrhage, in a surgical series of 54 giant pituitary adenomas, tumor hemorrhage developed in 3.7\% of patients after surgical resection [7]. In Goel's series of giant pituitary adenomas, on the other hand, tumor hemorrhage occurred in 3 patients among 118 tumors being treated by surgical resection. However, none of 21 patients who received radiation therapy as a part of initial management experienced apoplexy [8]. It is now considered that tumors of almost any size may undergo hemorrhage and apoplexy [9].

The relationship between cyst formation and the risk of hemorrhage has not been well discussed. It has been reported that pituitary cysts often represent radiological evidence of previous hemorrhage [10]. In the previously introduced Goel's series, 58 of 118 (49\%) giant pituitary adenomas exhibited cysts, 14 of these had accompanying fluid-fluid level formation, and 21 other cases had clots in the cysts that implied a recent event of hemorrhage into the cyst. Therefore, at least 35/58 (60\%) of cysts were related to the hemorrhage [8]. In the current series, the tumor was characterized by multiple cyst formation. Although fluid-fluid levels were not observed, the existence of multiple cysts might imply a history of repeated hemorrhage. It could therefore be speculated that the tumor in the current case was at higher risk of hemorrhage than ordinary pituitary adenoma, and hemorrhage could have developed regardless of the use of surgical intervention or radiation therapy.

\section{CONCLUSION}

Although radiotherapy with/without partial removal is generally accepted as a standard practice of GPAs, the risk of intratumoral hemorrhage must be better recognized, as this is a rare but life-threatening complication, even in ordinary non-functioning adenomas. The cystic components in pituitary adenoma could be a key pathogenesis of this rare event.

\section{CONFLICTS OF INTEREST NOTIFICATION}

None declared.

\section{FUNDING}

This study was partially supported by the Funding Program for World-Leading Innovative R\&D on Science and Technology (FIRST Program) initiated by the Coun- cil for Science and Technology Policy, as well as a Grant-in-Aid for Scientific Research (21591602) by the Japanese Society for the Promotion of Science.

\section{REFERENCES}

[1] de PaivaNeto, M.A., Vandergrift, A., Fatemi, N., Gorgulho, A.A., Desalles, A.A., Cohan, P., Wang, C., Swerdloff, R. and Kelly, D.F. (200) Endonasaltranssphenoidal surgery and multimodality treatment for giant pituitary adenomas. Clinical Endocrinology, 72, 512-519. http://dx.doi.org/10.1111/j.1365-2265.2009.03665.x

[2] Grigsby, P.W., Simpson, J.R., Stokes, S., Marks, J.E. and Fineberg, B. (1988) Results of surgery and irradiation or irradiation alone for pituitary adenomas. Journal of Neuro-Oncology, 6, 129-134. http://dx.doi.org/10.1007/BF02327388

[3] Lee, J.S., Park, Y.S., Kwon, J.T., Nam, T.K., Lee, T.J. and Kim, J.K. (2011) Radiological apoplexy and its correlation with acute clinical presentation, angiogenesis and tumor microvascular density in pituitary adenomas. Journal of Korean Neurosurgical Society, 50, 281-287.

[4] Liu, Z.H., Tu, P.H., Pai, P.C., et al. (2012) Predisposing factors of pituitary hemorrhage. European Journal of Neurology, 19, 733-738. http://dx.doi.org/10.1111/j.1468-1331.2011.03619.x

[5] Cardoso, E.R. and Peterson, E.W. (1984) Pituitary apoplexy: A review. Neurosurgery, 14, 363-373. http://dx.doi.org/10.1227/00006123-198403000-00021

[6] Jin, K.Y., Hyun, K.C., Hwan, C.J. and Min, K.J. (2011) Relationship between expression of vascular endothelial growth factor and intratumoral hemorrhage in human pituitary adenomas. Tumori, 97, 639-646.

[7] Koutourousiou, M., Gardner, P.A., Fernandez-Miranda, J.C., Paluzzi, A, Wang, E.W. and Snyderman, C.H. (2013) Endoscopic endonasal surgery for giant pituitary adenomas: Advantages and limitations. Journal of Neurosurgery, 118, 621-631. http://dx.doi.org/10.3171/2012.11.JNS121190

[8] Goel, A., Nadkarni, T., Muzumdar, D., Desai, K., Phalke, U. and Sharma, P. (2004) Giant pituitary tumors: A study based on surgical treatment of 118 cases. Surgical Neurology, 161, 436-446.

http://dx.doi.org/10.1016/j.surneu.2003.08.036

[9] Biousse, V., Newman, N.J. and Oyesiku, N.M. (2001) Precipitating factors in pituitary apoplexy. Journal of Neurology, Neurosurgery \& Psychiatry, 71, 542-545. http://dx.doi.org/10.1136/jnnp.71.4.542

[10] Mohr, G. and Hardy, J. (1982) Hemorrhage, necrosis, and apoplexy in pituitary adenomas. Surgical Neurology, 18, 181-189. http://dx.doi.org/10.1016/0090-3019(82)90388-3 


\section{ABBREVIATIONS}

$\mathrm{CT}=$ Computed tomography;

MRI = Magnetic resonance image;

TCS = Transcranial surgery;

$\mathrm{RT}=$ Radiation therapy;

VEGF = Vascular endothelial growth factor. 